\section{Efetividade das Estratégias de Ensino no Desenvolvimento das Competências de Estudantes do Curso de Administração}

\section{Teaching Strategies Effectiveness In Administration Course Students" Competency Development}

\section{Resumo:}

O objetivo desta investigação é estruturar um modelo que avalie a efetividade das estratégias de ensino (tradicional, online e hibrido) utilizadas para o desenvolvimento das competências dos estudantes de graduação em administração, observando o perfil do estudante e o estilo de aprendizagem. A aprendizagem online, baseada no e-learning, tem desempenhado um papel fundamental no ensino e aprendizagem, que se torna cada vez mais popular não apenas em diferentes níveis escolares, mas também em várias instituições de educação superior em todo o mundo (Nedungadi \& Raman, 2012). Nesse contexto, a educação a distância emerge como uma oportunidade que pode ampliar as possibilidades de aprendizagem do estudante, implementando ensino totalmente a distância ou no modelo híbrido.

Palavras-Chave: Modelos de aprendizagem, e-learning, tradicional, on-line, híbrido.

\section{Abstract:}

The objective of this research is to structure a model that evaluates the effectiveness of teaching strategies (traditional, online and hybrid) used for the development of Administration course undergraduate students' skills, observing student's profile and his/her learning style. Online learning, based on e-learning, has played a key role in teaching and learning, which is becoming increasingly popular not only at
Prof. ${ }^{2}$ Katia Maria

Rocha de Lima

Doutoranda da Universidade Cruzeiro do Sul e Fundação Inaciana Padre de Saboia (FEI),

Email: katia.lima@ cruzeirodosul.edu.br.

Área de doutoramento Administração - Área de Concentração - Gestão da Inovação - Linha de Pesquisa - Capacidades Organizacionais.

Orientador - Prof. Dr.

Edmilson Alves de Moraes Pesquisa com ou sem fomento - CAPES 
different levels of education but also in several higher education institutions around the world (Nedungadi \& Raman, 2012). In this context, distance education emerges as an opportunity that can broaden students' learning possibilities, implementing distance education or the hybrid model.

\section{Introdução}

O século XXI nos apresenta mudanças nas organizações e no ambiente competitivo. Na educação não é diferente, pois surge a necessidade de promover novas competências que suportem novos desafios destes futuros profissionais que ingressarão o mercado de trabalho. De um lado as instituições de ensino superior buscam transformar e moldar seus métodos de ensino para auxiliarem o aluno com um melhor currículo, neste ponto as novas tecnologias têm um papel importante (Ota, 2018). Nesse contexto, a educação a distância emerge como uma oportunidade que pode ampliar as possibilidades de aprendizagem do estudante, implementando ensino totalmente a distância ou no modelo híbrido. Com a demanda crescente por eventos educacionais como forma de atender novas exigências de desenvolvimento de competências individuais e organizacionais nas instituições de ensino e nas organizações de trabalho, as modalidades de entrega e metodologias de ensino estão sendo diversificadas, destacando-se as ações educacionais a distância mediada por novas tecnologias da informação e comunicação TICs (Martins, 2012). Em conjunto com o avanço da tecnologia da informação, grande parte dos sistemas de aprendizagem e os materiais de aprendizagem foram concebidos para o estudo individual online (Wang, 2014).

A aprendizagem online baseada no e-learning tem desempenhado um papel fundamental no ensino e aprendizagem, que se torna cada vez mais popular não apenas em diferentes níveis escolares, mas também em várias instituições de educação superior em todo o mundo (Nedungadi \& Raman, 2012).
O resultado, é um grande número de materiais online em diferentes áreas do conhecimento (Chao \& Chen, 2009; Wang, 2011). Contudo, parece confuso para os estudantes realizar de maneira eficaz a seleção do material didático de aprendizagem adequado a partir de dados disponíveis em larga escala de opções de e-learning. (REN, Yi et al., 2017).

A fim de desenvolver a capacidade de aprendizagem independente dos estudantes e melhorar seu desempenho e-learning, muitos estudos foram realizados. Um dos aspectos essenciais desses estudos é (Yu, Liao e Su, 2013; Yueh, Chen, Lin e Sheen, 2014) a aplicação de elementos multimídia abundantes em materiais de aprendizagem para facilitar o aprendizado de forma mais agradável (Yueh et al., 2014).

Há outros aspectos da pesquisa que focam em melhorar a interação on-line e orientação entre ensino e aprendizagem (Barra, Herrera, Cano e Vives, 2014; Sanchez-Torrubia, Torres-Blanc e Trivino, 2012; Wang, 2011). A maioria dos pesquisadores usam vários métodos de avaliação para observar os aspectos e melhorar a interação on-line e orientação entre ensino e aprendizagem (Barra, Herrera, Cano e Vives, 2014); (Sanchez-Torrubia, Torres-Blanc e Trivino, 2012; Wang, 2011). Eles utilizam vários métodos de avaliação para determinar e avaliar os fatores que impactam a eficácia do e-learning. Além disso, a inteligência artificial e a tecnologia de mineração de dados também oferecem novas formas de melhorar a eficácia da educação on-line (Blagojevic \& Micic, 2013).

Apesar da crescente atenção para a melhoria da qualidade dos serviços de cursos on-line, poucos trabalhos discutiram como ajudar os alunos a escolher o curso mais apropriado frente aos numerosos materiais de aprendizagem on-line. Com o desenvolvimento do e-learning, há cada vez mais educadores comprometidos com o desenvolvimento de cursos on-line (Castillo-Merino e Serradell-Lopez, 2014). Muitos materiais de e-learning com diferentes estilos de ensino e estratégias sobre assuntos semelhantes são acessíveis para os alunos. Obviamente, nem todo curso on-line seria aplicável a todos 
os alunos. De acordo com a literatura, o efeito do e-learning deve ser consideravelmente impactado pelas características dos estudantes, como a aprendizagem estilos de vida (Deborah, Baskaran, \& Kannan, 2014; Kolb \& Kolb, 2005), motivação de aprendizagem (Goda et al., 2015; Pintrich, 1999; Wang, 2011), tipos comportamentais de aprendizagem (Chang \& Chu, 2010; Goda et al., 2015; Wang, 2011) e conhecimento prévio (Kopcha \& Sullivan, 2008; Wang, 2010).

A promoção da aquisição de aprendizagem, nos cursos não presenciais ou semipresenciais, acompanha as transformações ocorridas no campo social e tecnológico, que surge como alternativa permitindo o atendimento de um número maior de estudantes, otimizando o tempo e reduzindo os gastos a médio e longo prazos (Zerbini, 2007). Essas mudanças recentes no campo da educação e do trabalho mostram a busca por novas e mais eficientes opções para fazer frente às exigências do mercado de trabalho por qualificação profissional, explorando situações que precisam ser discutidas. (Martins, 2012).

No Brasil, o tema Educação a Distância (EAD) tem se destacado no tocante às políticas públicas, através de ações e programas de governo para ampliação do acesso ao ensino por meio da modalidade, acompanhadas por alterações substanciais na legislação educacional. (Martins, 2012). A oferta de cursos de graduação a distância tem crescido em todo o país e ocorre em conjunto com os estudos que trazem o questionamento sobre a qualidade, efetividade e eficácia dos cursos nesta modalidade. Assim, faz-se necessário as discussões sobre as possibilidades e os desafios colocados pelas inovações em práticas de ensino-aprendizagem.

O novo instrumento de avaliação de Cursos de Graduação - Reconhecimento e Renovação (Presencial e a Distância), Nota Técnica $n^{\circ} 16 / 2017 / C G A C G I E S / D A E S$ e a Portaria n²0/2017 - demonstra a formulação de políticas nacionais e o aparecimento de documentos oficiais repensando práticas educacionais que possibilitem uma maior democratização do ensino, indicam para uma oportuna ocasião de se discutir e investigar a EAD como uma das modalidades educacionais disponíveis, especificamente para o nível superior de ensino, o que corrobora com as ações educacionais ofertadas a distância.

Nesse sentido, tornam-se necessárias investigações que testem a eficácia e aplicabilidade dessas ações educacionais, visando apreender quais são os processos de aprendizagem subjacentes envolvidos e os fatores influentes, de modo a garantir que os resultados pretendidos sejam atingidos. $O$ interesse de pesquisa na área de avaliação de procedimentos instrucionais a distância atinge grande relevância, pois acompanha as transformações que os cursos abertos, corporativos e ensino superior apresentam ao utilizarem a internet e as TICs.

As pesquisas científicas podem auxiliar no planejamento e avaliação de ações educacionais a distância, presencial ou hibrido, proporcionando uma reflexão às instituições de ensino superior que necessitam, muitas vezes, de orientação especializada para a implementação e acompanhamento dos cursos EaD ou híbridos. Deste modo, a reduzida sistematização de resultados de aprendizagem atingidos por ações dessa natureza (Carvalho \& Abbad, 2006; Castro \& Ferreira, 2006) pode ser explicada pelo surgimento e adoção recente desse tipo de processo educacional no país.

Esta pesquisa tem como objetivo propor e testar um modelo de análise que avalie a efetividade das estratégias de ensino (tradicional, online e hibrido) utilizadas para o desenvolvimento das competências dos estudantes de graduação em administração, observando o perfil do estudante e o estilo de aprendizagem.

Serão estudados variáveis antecedentes relacionadas ao perfil do estudante (Samarakou, M., Tsaganou, G., Papadakis, A, 2018; Broadbent, J., Fuller-Tyszkiewicz, M., 2018; Nakayama, M., Mutsuura, K., Yamamoto, H, 2017), estilos de aprendizagem (Alzain, A., et. al., 2017; Awang, H., et. al., 2017; Bouckenooghe, D., et. al., 2016.), efetividade nas estratégias de ensino (Rajab, K.D, 2018; Sheshasaayee, A., Nazreen 
Bee, M, 2018; Caporarello, L., Manzoni, B., Bigi, M.E, 2018; Tempelaar, D., et. al. 2018) e, por fim, as competências do século XXI ao associar as contribuições dos estudos relacionados a grupos de domínio cognitivo apresentado pelo National Research Council (2012) e de competências de conhecimentos fundamentais do World Economic Forum (Scott, 2015), que podem possibilitar o desenvolvimento de pesquisas diante das evidências que o bom desempenho nesses grupos de conhecimento são reflexo dos melhores resultados posteriores na vida do estudante.

Figura 1 - Proposição do modelo adaptado Modelo de Toulmin

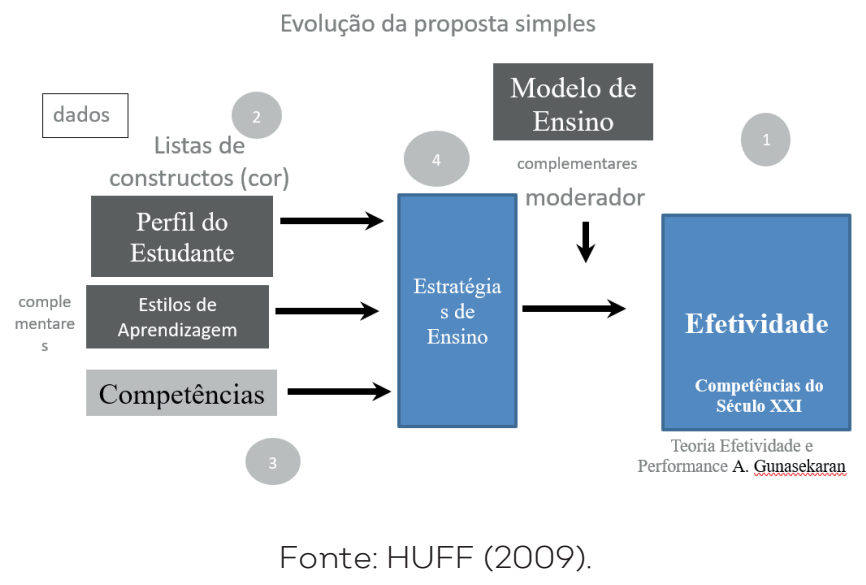

Por fim, faz-se necessário observar os estilos de aprendizagem e estratégias de ensino do curso de administração e as estratégias de ensino poderão corresponder às características dos estudantes, bem como se os estudantes melhoram seu desempenho no estudo. Daí uma avaliação de aplicabilidade projetado para que os alunos selecionem o curso e modalidade mais adequado com antecedência a fim de melhorar a efetividade de suas competências para atuação no mercado de trabalho. Para facilitar o acompanhamento da investigação, a Figura 1, conforme o modelo de Toulmin, demonstra a proposição do modelo que poderá sofrer ajustes no percurso de elaboração da tese.

A argumentação principal é a proposição de um modelo de análise que observe se há a formação de profissionais competentes, reflexivos, capazes de tomar decisões complexas e de basear-se em conhecimento pautado nas exigências do ambiente competitivo cada vez mais premente na atualidade. Nesse contexto, 0 desenvolvimento dos conhecimentos, habilidades e atitudes tem se tornado um pré-requisito para uma prática profissional eficaz. Desvendar as estratégias de ensino mais adequadas considerando o perfil do aluno, os estilos de aprendizagem que levem o estudante a atingir as competências do século XXI.

O Pressuposto conceitual desta investigação é - dependendo do perfil do estudante, do estilo de aprendizagem e da estratégia de ensino, o estudante atinge as competências necessárias, conforme representação na Figura 2:

Figura 2 - Pressuposto Conceitual adaptado Modelo de Toulmin



suposição contextual (escopo): na sala de estudo Fonte: HUFF (2009).

\section{Modelo de avaliação das estratégias de ensino: tradicional, on-line e hibrido}

O objetivo desta investigação é estruturar um modelo que avalie a efetividade das estratégias de ensino (tradicional, online e hibrido) utilizadas para o desenvolvimento das competências dos estudantes de graduação em administração, observando o perfil do estudante e o estilo de aprendizagem. Para os objetivos específicos, propõe-se:

- Identificar os diferentes perfis de estudantes do curso de administração, por meio de uma Revisão de Literatura, a 
fim de reunir contribuições para o contexto de estratégias de aprendizagem no ensino superior.

- Identificar os diferentes estilos de aprendizagem dos estudantes do curso de administração, por meio de uma Revisão de Literatura, a fim de reunir contribuições para o contexto de estratégias de aprendizagem no ensino superior.

- Validar um modelo que avalie a efetividade das estratégias de ensino, buscando as competências do século XXI em estudantes do curso de Administração, tradicionais, online ou híbrido.

Nesse contexto, o desenvolvimento desta tese está condicionado à seguinte pergunta: quais são os fatores que impactam na efetividade do desenvolvimento das competências do estudante de administração? A hipótese sugerida é que, dependendo do perfil do aluno, estilo de aprendizagem e estratégia de ensino, poderá influenciar a efetividade da aquisição das competências do século XXI.

Ao cumprir com esses objetivos, esta investigação pretende contribuir para personalização da aprendizagem dos estudantes de cursos do ensino superior no curso de Administração e futuramente replicar o modelo de análise para outras áreas do conhecimento.

\section{Método de avaliação dos modelos de avaliação das estratégias de ensino}

O Método utilizado, iniciamente, será a revisão sistemática da literatura nas seguintes bases de dados: Web of Science, SCOPUS, JCR, ERIC, JSTOR, Portal de Periódicos da CAPES, SAGE e em banco de dados de teses. A seleção inicial dos estudos avaliada bem como a qualidade metodológica será verificada nesta etapa da pesquida.

O público-alvo escolhido é os estudantes dos três modelos citados (Presencial, Cursos EaD e cursos EaD com encontros presenciais), tendo como fonte de coleta de dados o curso de Administração, sem restrição de etapa de matrícula. Sendo assim, a amostra será representada por estudantes de diferentes modalidades.

Para identificar quais são os fatores explicativos para a utilização das estratégias de aprendizagem utilizadas pelos estudantes de administração, busca-se os procedimentos documentais e utilização de dados provenientes de questionário aplicados aos estudantes a partir de escalas validadas.

A abordagem do estudo será a quantitativa, sendo utilizados procedimentos estatísticos para responder à questão de pesquisa. A utilização de técnicas multivariadas também serão utilizadas para testar o modelo proposto para analisar a efetividade das estratégias de ensino a partir do perfil e estilo de aprendizagem do estudante, observando se essas estratégias promovem o aprimoramento das competências do seculo XXI, ou seja, competências congnitivas, intrapessoal e interpessoal.

A coleta será realizada a partir da validacão do estudo na banca de qualificação da tese em 2019. Os questionários serão entregues em formulário eletrônico enviado por e-mail e fixado na plataforma do ambiente virtual já utilizado pelo estudante.

O instrumento de coleta de dados será formado por perguntas com o intuito de caracterizar o respondente, como gênero, idade, semestre, tempo de estudo, perguntas sobre estilos de aprendizagem e perfil do estudante e por perguntas que visavam identificar as estratégias de aprendizagem.

A viabilização de dados para essa investigação será a utilização de dados do Grupo Educacional Cruzeiro do Sul, que acreditamos ser uma proposta viável, tendo em vista a representatividade que esta instituição tem na cidade de São Paulo. Atualmente o grupo conta com mais de 250.000 alunos, distribuídos em cursos de graduação em diferentes áreas do conhecimento em todo o Brasil. 
A instituição escolhida possui três modalidades de oferta de cursos de graduação: Presencial, Online e Semipresencial.

1. Presencial com Disciplinas Online;

2. EaD apenas com avaliações presenciais;

3. EaD com encontros presenciais.

A proposta poderá satisfazer de maneira suficiente os critérios oferecidos por Castro (1978), tais como: importância, originalidade e viabilidade, e acredita-se que esta investigação se justifica. $O$ modelo é apresentado na Figura 3, que traz a contribuição teórica do estudo.

Figura 3 - Modelo Proposto de efetividade

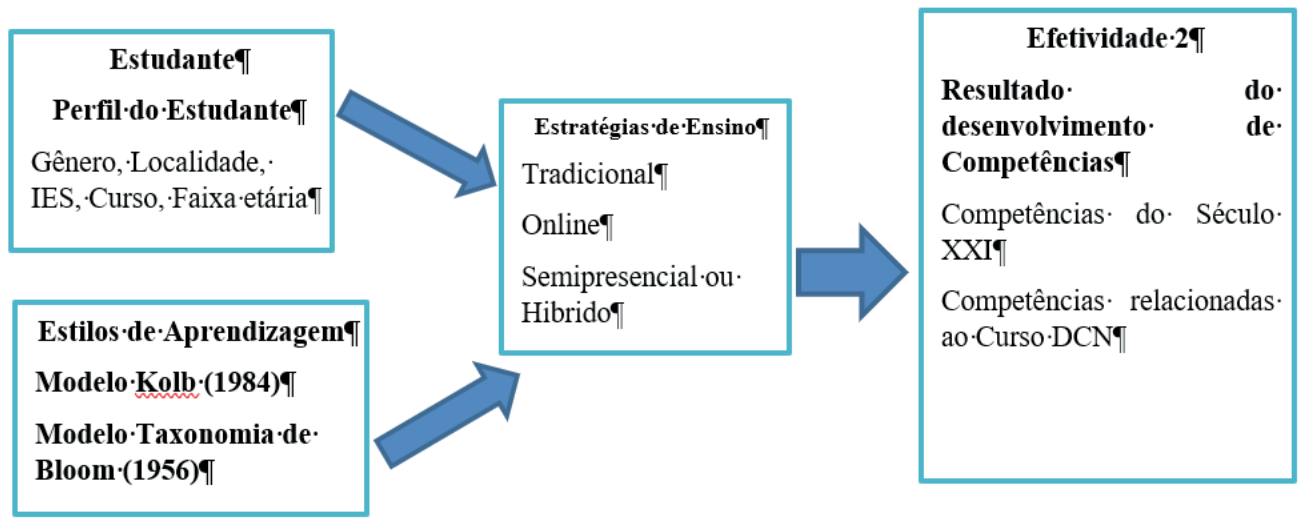

O bubble map do tema de pesquisa e identificação dos diálogos de pesquisa. Lista dos principais autores e os journals que formam esse diálogo.

Mapeamento SCOPUS - Geral - E-learning, Blended Learning, Efetividade, Aprendizagem, Perfil do aluno, Estilo de Aprendizagem e Teorias de Aprendizagem. Mapeamento 5 - Geral - E-learning, Blended Learning, Efetividade, Aprendizagem, Perfil do aluno, Estilo de Aprendizagem e Teorias de Aprendizagem. Ocorrências
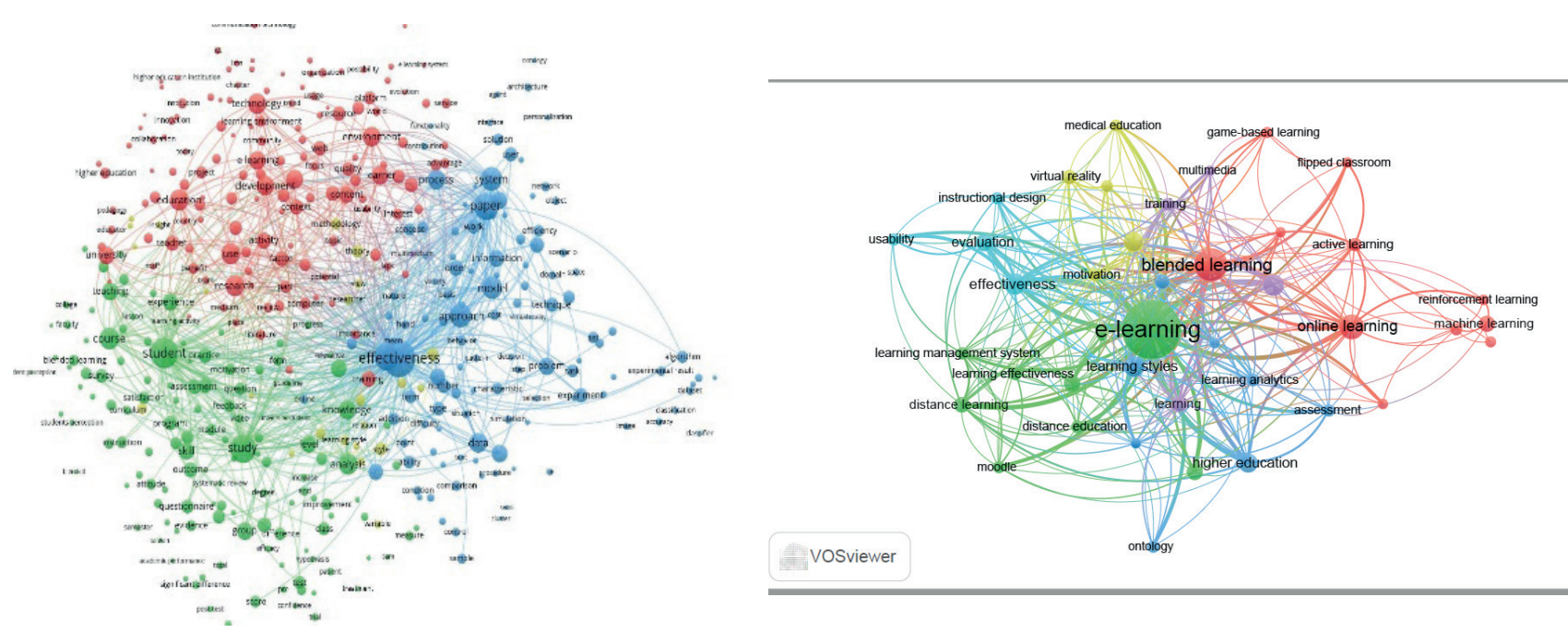
Mapeamento 6 - Geral - E-learning, Blended Learning, Efetividade, Aprendizagem, Perfil do aluno, Estilo de Aprendizagem e Teorias de Aprendizagem. Autores Mapeamento 7 -- E-learning, Efetividade
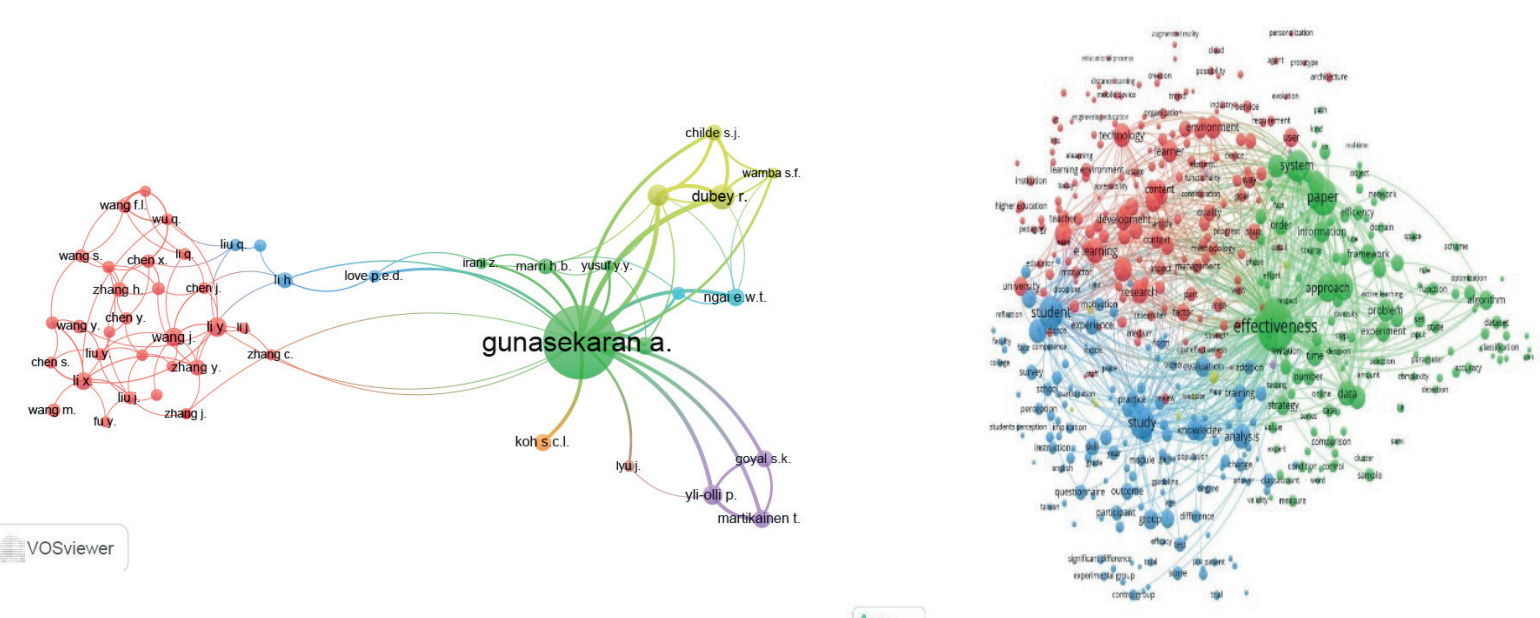

\section{Ansoserer}

Mapeamento 8 -- E-learning, Efetividade - Autores/

Mapeamento 9 -- Blended-learning, Efetividade - Ocorrências e autores
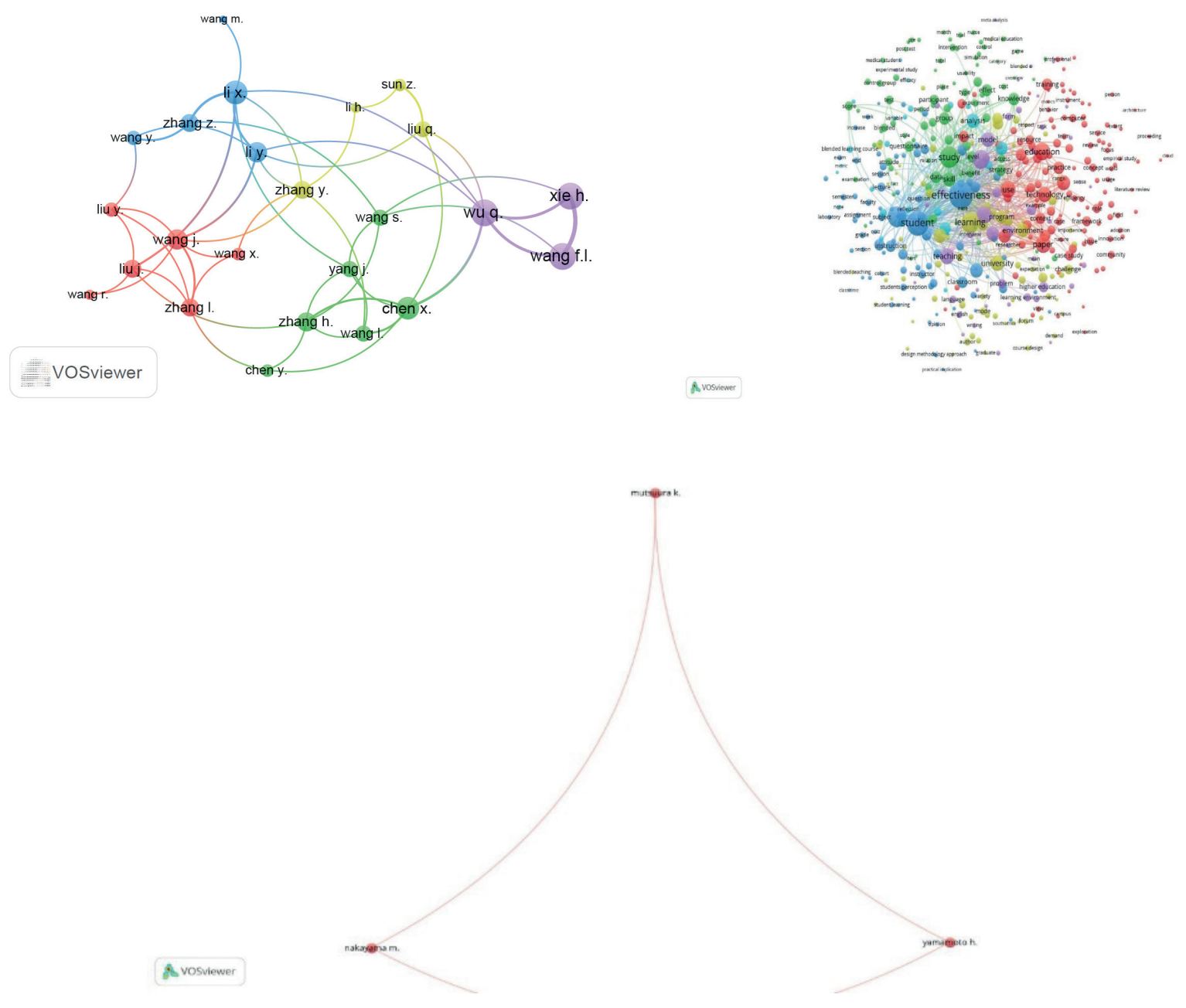


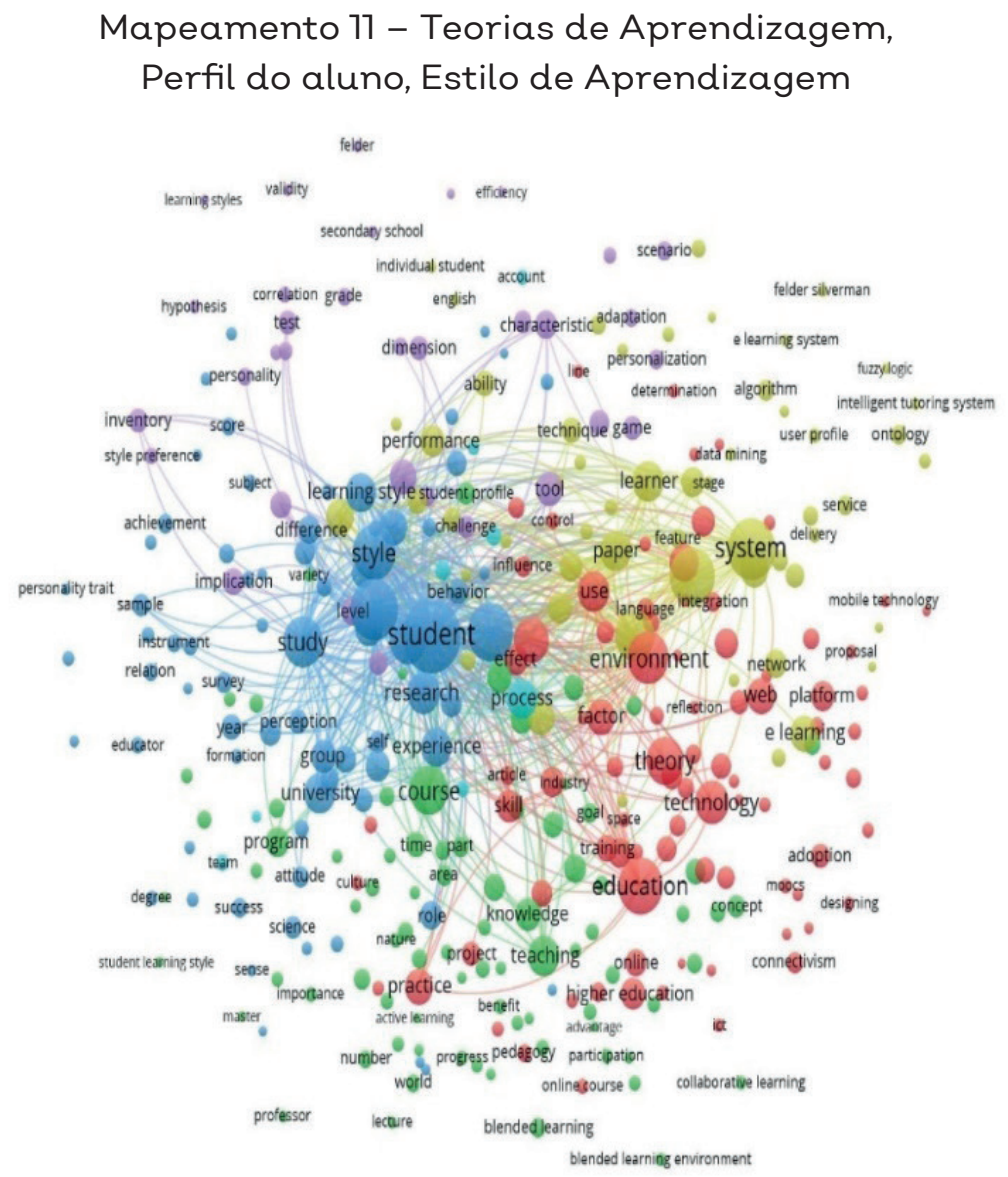

B vosviewer

Mapeamento 12 - Teorias de Aprendizagem, Perfil do aluno, Estilo de Aprendizagem - Autores

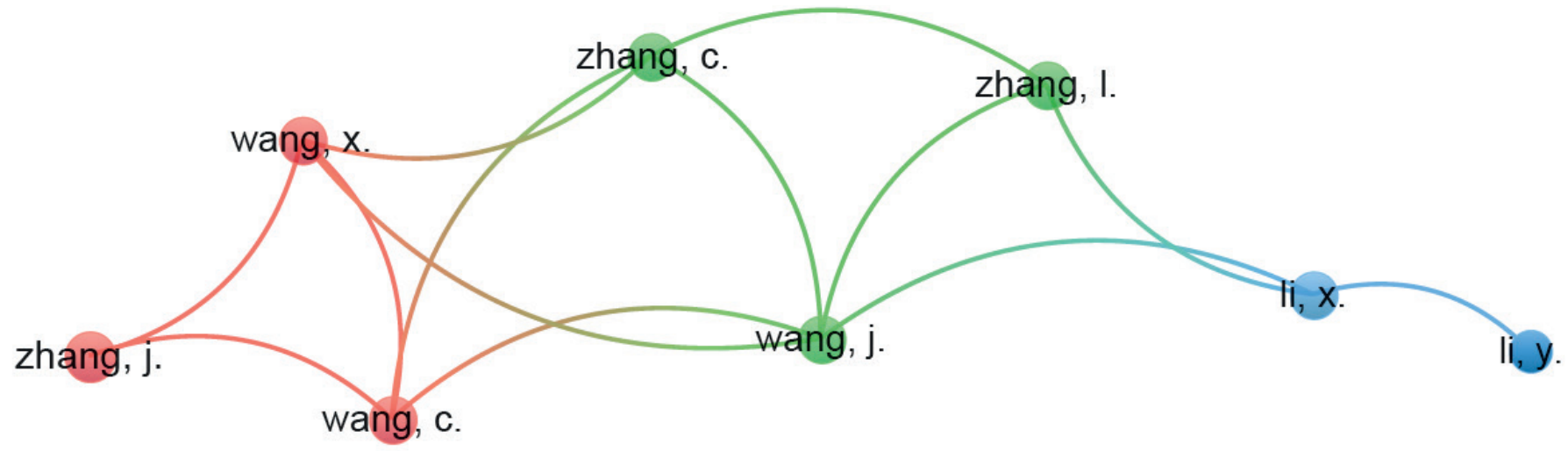

\section{VOSviewer}


Mapeamento 13 - Perfil do aluno,

Estilo de Aprendizagem

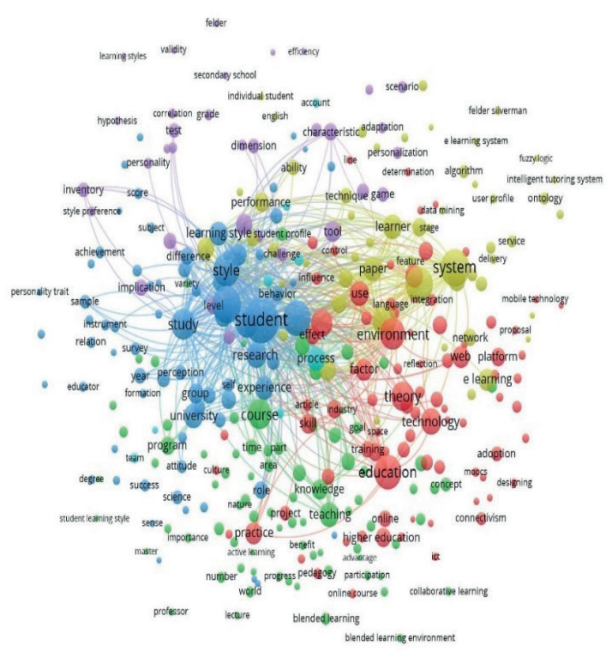

Mapeamento 14 - Perfil do aluno, Estilo de Aprendizagem - Autores

VOSviewer

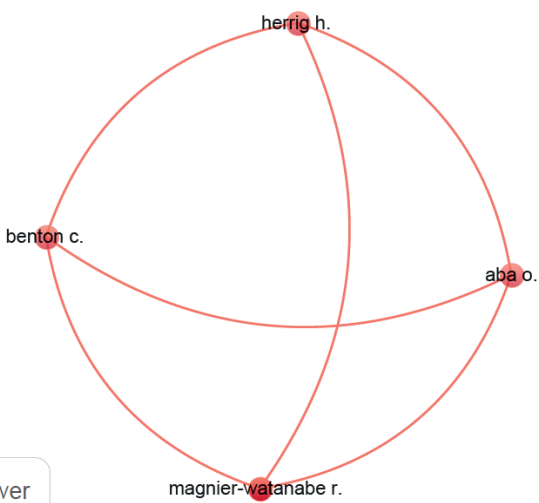

\section{Considerações Finais}

A pesquisa poderá contribuir com explicações relacionadas ao impacto de variáveis dependentes como o perfil do estudante e estilo de aprendizagem, atrelado a (variável moderadora), estratégias de ensino e se estas tem promovido as competências do século XXI (variável independente).

Como resultado, espera-se a criação de um modelo que contemple o perfil do aluno, os estilos de aprendizagem, as estatégias de ensino (tradicional, online ou hibrido) e como estes impactam na melhoria da efetividade das es- tratégias de ensino focadas na aprendizagem personalizada ou adaptativa.

Estratégias de ensino personalizadas é uma tendência apontada pelo último relatório do NMC Horizon Report - 2017 Higher Education Edition (BECKER, et al., 2017), que destacou: o uso das tecnologias educacionais em aceleração de curto a médio prazo, como o design de modelos de ensino híbrido, observando-se o crescimento da oferta do ensino online e híbrido que complementam as atividades tradicionais de sala de aula nas IES; a aprendizagem colaborativa, que oferece ganhos sociais, emocionais e de aprendizado, além de ferramentas baseadas em nuvem, aplicativos e espaços de trabalho compartilhados que permitem que os educadores promovam pedagogias que se centram no aprendizado peer-to-peer (aprendizagem por pares) e grupo; os espaços de aprendizagem para suportar as melhorias de novas formas de ensino e aprendizagem; e, por fim, as estratégias para medir a aprendizagem. (BECKER, S. A. et al., 2017).

Neste estudo, dentre as tecnologias educacionais apontadas como emergentes e com potencial para impulsionar mudanças na educação nos próximos cinco anos, destacam-se três para corroborar com esta tese: como as tecnologias de aprendizagem adaptativa; a "Next-Generation" dos ambientes de gestão da aprendizagem, do inglês, Learning Management System (LMS), em que esses novos modelos de sistemas de gerenciamento de aprendizagem podem se tornar mais flexíveis e melhor suportar ambientes de aprendizagem personalizados; e a Inteligência Artificial, criar máquinas inteligentes que imitam o comportamento humano podem reforçar a produtividade, o engajamento de estudantes e oferecer experiências de aprendizagem personalizadas.

Assim, essa tese poderá contribuir com a criação de um modelo e análise seguido do teste prático para observar e explicar os impactos das estratégias de ensino, baseadas no estilo de aprendizagem e perfil do estudante e se estas promovem de fato as competências do século XXI. 


\section{Referências}

ALZAIN, Alzain et al. LAES: An adaptive education system based on learners' learning styles. In: Knowledge Engineering and Applications (ICKEA), 2017 2nd International Conference on. IEEE, p. 107-111, 2017.

AWANG, H. et al. Relationship between the Learning Styles Preferences and Academic Achievement. In: IOP Conference Series: Materials Science and Engineering. IOP Publishing. p. 012193, 2017.

BARRA, Enrique et al. Using multimedia and peer assessment to promote collaborative e-learning. New Review of Hypermedia and Multimedia, v. 20, n. 2, p. 103-121, 2014

BECKER, S. A. et al. NMC horizon report: 2017 higher education edition. Austin, Texas: The New Media Consortium, 2017.

BLAGOJEVIĆ, Marija; MICIĆ, Živadin. A webbased intelligent report e-learning system using data mining techniques. Computers \& Electrical Engineering, v. 39, n. 2, p. 465-474, 2013.

BOUCKENOOGHE, Dave et al. Exploring the impact of cognitive style profiles on different learning approaches: Empirical evidence for adopting a person-centered perspective. Learning and Individual Differences, v. 51, p. 299-306, 2016.

BRASIL. Inep. Nota técnica n ${ }^{\circ}$ 16/2017/CGACGIES/DAES, de 15 de dezembro de 2017, sobre os Novos instrumentos de avaliação externa: instrumento de avaliação institucional externa - Presencial e a distância (iaie); instrumento de avaliação de cursos de graduação - presencial e a distância (iacg). Disponível em: <http:// download.inep.gov.br/educacao_superior/avaliacao_cursos_graduacao/legislacao_normas/2017/nota_tecnica_sei_inep_0126132.pdf>. Acesso em: 15/06/2018.
BRASIL. Ministério da Educação. Portaria $n^{\circ}$ 20, de 21 de dezembro de 2017. Brasília, 2018. <https://goo.gl/uoeXLZ>

BRASIL. Sistema nacional de avaliação da educação superior (SINAES): Instrumento de Avaliação Institucional Externa Presencial e a distância. Disponível em: <http://download. inep.gov.br/educacao_superior/avaliacao_ institucional/instrumentos/2017/IES recredenciamento.pdf>. Acesso em: 15/06/2018. CAPORARELLO, Leonardo; MANZONI, Beatrice; BIGI, Martina. E-learning Effectiveness from a Students' Perspective: An Empirical Study. In: Digital Technology and Organizational Change. Springer, Cham, p. 163-172, 2018.

CARVALHO, Renata Silveira; ABBAD, Gardênia. Avaliação de treinamento a distância: reação, suporte à transferência e impactos no trabatho. Revista de Administração contemporânea, v. 10, n. 1, p. 95-116, 2006

CASTILLO-MERINO, David; SERRADELLLÓPEZ, Enric. An analysis of the determinants of students' performance in e-learning. Computers in Human Behavior, v. 30, p. 476484, 2014

CASTRO, Claudio de Moura. A Prática da Pesquisa. São Paulo: MCGraw-Hill do Brasil, 1978.

CASTRO, M. N. M., \& Ferreira, L. D. V. TD\&E a distância: múltiplas mídias e clientelas. In J. E. Borges-Andrade, G. Abbad, L. Mourão (Orgs.). Treinamento, desenvolvimento e educação em organizações e trabalho: fundamentos para a gestão de pessoas p. 322-339. Porto Alegre: Artmed, 2006.

CHANG, Yi-Chun; CHU, Chih-Ping. Applying learning behavioral Petri nets to the analysis of learning behavior in web-based learning environments. Information Sciences, v. 180, n. 6. p. 995-1009, 2010.

CHAO, Ru-Jen; CHEN, Yueh-Hsiang. Evaluation of the criteria and effectiveness of distance e-learning with consistent fuzzy preference relations. Expert Systems with Applications, v. 36, n. 7, p. 10657-10662, 2009. 
DEBORAH, L. Jegatha; BASKARAN, Ramachandran; KANNAN, Arputharaj. Learning styles assessment and theoretical origin in an E-learning scenario: a survey. Artificial Intelligence Review, v. 42, n. 4, p. 801-819, 2014.

GODA, Yoshiko et al. Procrastination and other learning behavioral types in e-learning and their relationship with learning outcomes. Learning and Individual Differences, v. 37, p. 72-80, 2015.

HUFF, Sigismund Anne. Designing Research for Publication. California: Sage Publications, 2009.

KOLB, Alice Y.; KOLB, David A. Learning styles and learning spaces: Enhancing experiential learning in higher education. Academy of management learning \& education, v. 4, n. 2, p. 193-212, 2005.

KOPCHA, Theodore J.; SULLIVAN, Howard. Learner preferences and prior knowledge in learner-controlled computer-based instruction. Educational Technology Research and Development, v. 56, n. 3, p. 265-286, 2008.

NAKAYAMA, Minoru; MUTSUURA, Kouichi; YAMAMOTO, Hiroh. Effectiveness of Student's Note-Taking Activities and Characteristics of Their Learning Performance in Two Types of Online Learning. International Journal of Distance Education Technologies (IJDET), v. 15, n. 3, p. 47-64, 2017.

NATIONAL RESEARCH COUNCIL. Education for Life and Work: Developing Transferable Knowledge and Skills in the 21st Century. Washington, DC: The National Academies Press, 2012.

NEDUNGADI, Prema; RAMAN, Raghu. A new approach to personalization: integrating e-learning and m-learning. Educational Technology Research and Development, v. 60, n. 4, p. 659-678, 2012.
OTA, Marcos Andrei. Adaptatividade em ambientes virtuais: uma proposta para personalizar a aprendizagem em cursos híbridos de ensino superior. 2018.

PINTRICH, Paul R. The role of motivation in promoting and sustaining self-regulated learning. International journal of educational research, v. 31, n. 6, p. 459-470, 1999.

RAJAB, Khairan D. The Effectiveness and Potential of E-Learning in War Zones: An Empirical Comparison of Face-to-Face and Online Education in Saudi Arabia. IEEE Access, v. 6, p. 6783-6794, 2018.

REN, Yi et al. Exploring an on-line course applicability assessment to assist learners in course selection and learning effectiveness improving in e-learning. Learning and Individual Differences, v. 60, p. 56-62, 2017.

SAMARAKOU, Maria; TSAGANOU, Grammatiki; PAPADAKIS, Andreas. An e-Learning System for Extracting Text Comprehension and Learning Style Characteristics. Journal of Educational Technology \& Society, v. 21, n. 1, p. 126-136, 2018.

SÁNCHEZ-TORRUBIA, M. Gloria; TORRESBLANC, Carmen; TRIVINO, Gracian. An approach to automatic learning assessment based on the computational theory of perceptions. Expert Systems with Applications, v. 39, n. 15, p. 12177-12191, 2012.

SCOTT, C. L. The Futures of Learning 1: Why must learning content and methods change in the 21st century? UNESCO Education Research and Foresight. Paris. [ERF Working Papers Series, No. 13], 2015.

SHESHASAAYEE, Ananthi; BEE, M. Nazreen. Analyzing Online Learning Effectiveness for Knowledge Society. In: Information Systems Design and Intelligent Applications. Springer, Singapore, 2018. p. 995-1002. 
TEMPELAAR, Dirk; RIENTIES, Bart; NGUYEN, Quan. Investigating learning strategies in a dispositional learning analytics context: the case of worked examples. In: Proceedings of the 8th International Conference on Learning Analytics and Knowledge. ACM, 2018. p. 201-205.

WANG, Tzu-Hua. Developing an assessmentcentered e-Learning system for improving student learning effectiveness. Computers \& Education, v. 73, p. 189-203, 2014.

WANG, Tzu-Hua. Developing Web-based assessment strategies for facilitating junior high school students to perform self-regulated learning in an e-Learning environment.
Computers \& Education, v. 57, n. 2, p. 18011812, 2011.

YU, Pao-Ta; LIAO, Yuan-Hsun; SU, MingHsiang. A near-reality approach to improve the e-learning open courseware. Educational Technology \& Society, v. 16, n. 4, p. 242-257, 2013.

YUEH, Hsiu-Ping et al. Developing digital courseware for a virtual nano-biotechnology laboratory: A design-based research approach. Journal of Educational Technology \& Society, v. 17, n. 2, 2014

ZERBINI, Thaís. Avaliação da transferência e treinamento em curso a distância. 2007. 